\title{
Preface to the Fifth Group of "Waves in Plasma" Papers
}

This issue of Radio Science contains the fifth and final grouping of papers on "Waves in Plasma." It is expected that individual papers on this subject will continue to be published in regular issues of the journal.

The first paper of the issue is a comprehensive treatment by $\mathrm{F}$. W. Crawford of resonance phenomena occurring at the harmonics of the cyclotron frequency. These effects would not occur in a cold plasma and thus the resonance "spikes" are not predicted by classical magneto-ionic theory. The results have important implications in both laboratory and ionospheric plasma physics.

The second paper, by R. L. Gallawa, also deals with a warm plasma problem. The author presents an analysis for an electroacoustic wave incident onto a plane interface between the plasma and free space. It is shown that a major part of the acoustic energy may be reradiated as electromagnetic energy into the free space region.

The third paper, by D. R. Croley, Jr., and B. S. Tanenbaum, is a useful, graphical presentation of the computed phase and attenuation characteristics of plane waves in a multicomponent plasma. The conditions are chosen to correspond to the 80,100 , and $300 \mathrm{~km}$ levels in the ionosphere for both longitudinal and transverse propagation over a frequency range from $10^{-5}$ to $10^{9} \mathrm{c} / \mathrm{s}$.

The fourth paper, by Dr. G. C. Reid, is an interesting analysis of the transfer of electrostatic fields from the outer magnetosphere to the ionosphere via the geomagnetic field lines. The results would appear to have relevance to the origin of ionospheric irregularities.

The fifth paper, by G. Tyras, P. C. Bargelioties, J. M. Hamm, and R. R. Schell, describes an ingenious technique to simulate the plasma sheath which surrounds a microwave antenna on a re-entry vehicle. The results compare quite favorably with calculations for radiation from a slot in a conducting ground plane which has a uniform (simulated) plasma coating.

The sixth paper, by S. N. Samaddar, considers the influence of the orientation of the external magnetic field on the characteristics of the plasma sheath. The results have possible application to re-entry communications during the blackout phase.

The seventh and final "Waves in Plasma" paper is a contribution, from the editor, which deals with the mechanism underlying reflection of waves in inhomogeneous plasma. The principal conclusion is that reflection does not take place at a discrete level in the medium.

In the selection and review of the "Waves in Plasma" papers in the past five issues, the editor has had valuable assistance from a number of individuals. The names of these people are listed as follows, along with the number of papers they have reviewed: S. Adachi (1), W. S. Ament (1), M. P. Bachynski (2), K. Balmain (1), R. E. Barrington (1), Petr Beckmann (1), W. E. Blair (1), H. G. Booker (2), F. X. Bostick (1), K. L. Bowles (4), J. Brandstatter (1), S. J. Buchsbaum (1), K. G. Budden (1), O. Buneman (2), R. E. Burgess (1), Paul Caron (1), K. M. Chen (2), M. Chodorow (1), M. H. Cohen (1), F. W. Crawford (1), A. L. Cullen (2), K. Davies (2), R. S. Elliott (1), M. Epstein (1), W. C. Erickson (1), A. J. Estin (1), D. T. Farley, Jr. (1), F. C. Fehsenfeld (1), J. A. Fejer (1), L. P. Felsen (6), W. L. Flock (2), J. Galejs (2), R. L. Gallawa (1), O. Garriott (1), S. H. Gross (1), A. Hessel (2), H. Hodara (1), A. Ishimaru (2), J. R. Johler (1), Eric Johnson (1), R. M. Jones (1), T. Kahan (1), M. Katzin (1), R. W. P. King (1), R. S. Lawrence (3), L. A. Manning (1), Keith McLane (2), L. R. Megill (1), R. Mittra (1), A. A. Oliner (2), Leon Peters (1), H. Poeverlein (1), R. S. Powers (2), G. H. Price (1), J. M. Richardson (1), W. Rotman (2), R. V. Row (2), P. Schlesinger (1), S. R. Seshadri (1), J. Shmoys (1), K. M. Siegel (1), R. L. Smith (1), R. N. Sudan (1), C. T. Tai (1), H. Unz (1), and B. Wieder (1). The editor would also like to thank Mrs. Mildred Talbutt and Mrs. Dorothy Burdick for their untiring efforts in preparing these papers for the NBS printing section.

JAMES R. WAIT, Editor 